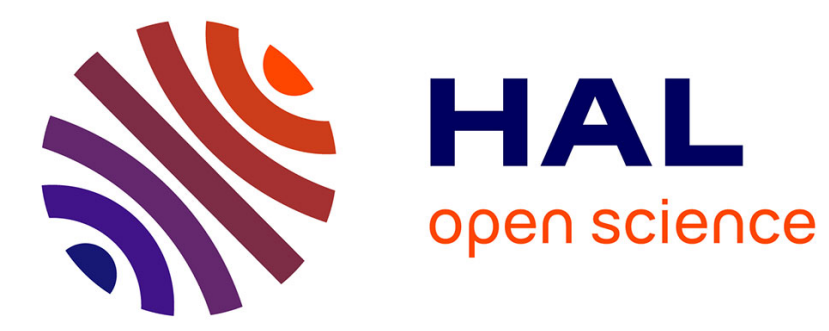

\title{
Noi e gli altri, l'enjeu migratoire, miroir de la crise politique italienne?
}

Évelyne Ritaine

\section{To cite this version:}

Évelyne Ritaine. Noi e gli altri, l'enjeu migratoire, miroir de la crise politique italienne?. Pôle Sud, 1999, Enjeux migartoires en Europe du Sud, 11 (11), pp.55-69. 10.3406/pole.1999.1051 . halshs00105430

\section{HAL Id: halshs-00105430 \\ https://shs.hal.science/halshs-00105430}

Submitted on 9 May 2019

HAL is a multi-disciplinary open access archive for the deposit and dissemination of scientific research documents, whether they are published or not. The documents may come from teaching and research institutions in France or abroad, or from public or private research centers.
L'archive ouverte pluridisciplinaire HAL, est destinée au dépôt et à la diffusion de documents scientifiques de niveau recherche, publiés ou non, émanant des établissements d'enseignement et de recherche français ou étrangers, des laboratoires publics ou privés. 


\section{Noi e gli altri, l'enjeu migratoire, miroir de la crise politique italienne} Mme Evelyne Ritaine

\section{Citer ce document / Cite this document :}

Ritaine Evelyne. Noi e gli altri, l'enjeu migratoire, miroir de la crise politique italienne. In: Pôle Sud, n¹1, 1999. Enjeux migratoires en Europe du Sud. pp. 55-69;

doi : https://doi.org/10.3406/pole.1999.1051

https://www.persee.fr/doc/pole_1262-1676_1999_num_11_1_1051

Fichier pdf généré le 23/04/2018 


\begin{abstract}
Within southern Europe, the specificity of the Italian approach to immigration is its degree ofpoliticisation. This has also taken a specific course : not only is there the usual balancing act between repressive necessities and commitments to democracy, but, through certain political parties, there has been a naturalisation of xenophobic themes which has taken the form of equating insecurity with immigration. The dynamics of this positionning, both in terms of inter-party relationships and in civil society, is caught up in the ongoing recomposition of Italian politics. More than in other countries, this context has made the effects of confronting the Other even stronger on representations of the nation, the state, nationality and citizenship, concepts which are all at the heart of the Italian political crisis.
\end{abstract}

\title{
Résumé
}

L'originalité de l'Italie, dans l'ensemble sud-européen, réside tout d'abord dans la politisation du thème de l'immigration. Cette politisation se réalise aussi dans un sens spécifique : non seulement on y retrouve le balancement habituel entre nécessités répressives et volontés démocratiques, mais encore voit-on s y réaliser, par l'entremise de certains partis politiques, une "naturalisation politique" des thèmes xénophobes, sous la forme d'une équivalence symbolique entre insécurité et immigration. La dynamique des prises de position, dans les rapports interpartisans et dans la société civile, est prise dans le mouvement de la recomposition politique italienne. Ce contexte rend, plus qu'ailleurs, sensibles les effets de la confrontation à l'altérité sur les représentations de la Nation et de l'État, de la nationalité et de la citoyenneté, qui sont au principe de la crise politique italienne. 


\title{
Noi e gli altri, l'enjeu migratoire, miroir de la crise politique italienne
}

\author{
par Évelyne Ritaine \\ FNSP/CERVL-IEP Bordeaux \\ Pôle Sud $N^{\circ} 11$ - novembre $1999-p .55$ d 69
}

Pourquoi, à un moment donné, la question de l'immigration devient-elle un enjeu politique, non seulement problème susceptible de traitement public, mais aussi objet de luttes partisanes et thème de mobilisations sociales? Le sens commun prétend aisément que l'accentuation de la pression migratoire est à l'origine de cette émergence dans l'arène publique. Or, dans le cas de l'Italie, les flux d'immigration se sont intensifiés avec régularité, depuis la fin des années 70, dans l'indifférence publique et dans une relative tolérance sociale, du moins jusqu'au début des années 90. De plus la faible ampleur du phénomène, les étrangers ne représentant pas plus de $2 \%$ de la population résidente, n'autorise pas à prendre cette explication mécanique au sérieux. Un argument plus élaboré est parfois avancé, qui invoque l'effet de la construction européenne, en l'occurrence l'ouverture d'un espace de libre-circulation intracommunautaire. La volonté italienne de faire partie de l'espace défini par les accords de Schengen, qui a instauré des obligations de contrôle du territoire rendues encore plus évidentes ensuite par leur intégration dans le Traité d'Amsterdam, les difficultés qu'a rencontrées ce pays à être intégré dans l'espace de libre-circulation, auraient ainsi contribué à l'inscription du thème migratoire dans les débats publics'. Ce second facteur explicatif, quoique plus pertinent, a cependant une portée limitée : en effet, même si l'existence de normes européennes oblige les pouvoirs publics à un travail de mise en conformité, donc à une inscription sur l'agenda politique, dans de nombreux pays, et en Italie jusqu'en 1990, ce travail s'effectue à bas bruit, dans les cercles gouvernementaux et dans les réseaux d'experts. Ni les grands partis politiques, ni les spécialistes de la bureaucratie publique n'ont tendance à débattre de cette question potentiellement sensible dans l'arène publique. Pour qu'il y ait politisation du thème, il semble bien qu'il faille que des acteurs politiques entreprennent d'utiliser celui-ci comme une ressource dans leurs stratégies politiques, contraignant ainsi leurs adversaires politiques, puis par contamination tous les acteurs sociaux concernés, à prendre position : ce processus de politisation intervient d'autant plus facilement que les forces politiques sont plus fragmentées, donc les stratégies de distinction entre partis plus nécessaires, et qu'une crise politique majeure bouscule tous les rapports de force, fragmentation et crise caractéristiques de la situation italienne dans les années 90. Loriginalité de l'Italie, dans l'ensemble sud-européen, réside tout d'abord dans cette politisation du thème. Elle la réalise aussi dans un sens spécifique : non seulement on y retrouve le balancement entre nécessités répressives et volontés démocratiques, classique dans les différents pays européens et au sein même des normes de l'Union, mais encore voit-on s'y réaliser, par l'entremise de certains partis politiques, une "naturalisation politique" des thèmes 


\section{Pôle Sud $N^{\circ} 11$}

xénophobes, et, par contrecoup, une radicalisation de la mobilisation antiraciste. Cependant, si ce processus de politisation dans l'arène partisane est le plus apparent, et s'il représente sans doute l'armature du cadre cognitif des politiques concernant l'immigration, il n'est que la partie émergée d'un processus de production des représentations politiques bien plus complexe. À l'analyse en effet, les prises de position des acteurs politiques se révèlent informées et influencées par de multiples acteurs sociaux (représentations d'intérêt, associations du "troisième secteur", experts de la bureaucratie publique), qui apparaissent comme le véritable creuset de la production politique sur ces thèmes. La logique de leurs engagements dessine un espace de médiation politique spécifique à la question de l'immigration, fait de l'entrecroisement de multiples réseaux d'échange politique. La dynamique de ces prises de position, dans les rapports interpartisans et dans la société civile, est prise dans le mouvement de la recomposition politique italienne. $\mathrm{Ce}$ contexte rend, plus qu'ailleurs, sensibles les effets de la confrontation à l'altérité (noi e gli altri : nous et les autres) sur les représentations de la Nation et de l'État, de la nationalité et de la citoyenneté : celles-ci ne sont-elles pas au principe de la crise politique italienne?

\section{Le temps d'une non-politique publique : au péril de la xénophobie}

La généalogie de la législation italienne en matière migratoire est l'histoire de la quête difficile d'un référentiel national, sous la pression de l'impératif européen d'une part, de l'exploitation xénophobe du thème par certains partis d'autre part. Trois grandes dates jalonnent ce parcours, la loi de 1990 dite loi Martelli, le décret-loi du gouvernement Dini en 1995, et la récente loi de 1998 dite loi Turco-Napolitano : au travers des évolutions qui s'y manifestent sont visibles aussi bien le processus d'instrumentalisation du thème par des stratégies de distinction politique entre les partis que le poids de l'influence de certains groupes de pression. Si le premier processus est un facteur de radicalisation des interprétations, le second, en ménageant de nombreux espaces de négociation et d'échange politique, pousse à la recherche de solutions consensuelles, tant symboliques que pratiques.

Dans ce pays dont la mémoire collective est si marquée par l'émigration (Ritaine, 1998), il est symptomatique de la non-visibilité des nouveaux problèmes que, jusqu'en 1986, le statut des étrangers n'ait été réglé que par un texte de 1931, le Testo unico di pubblica sicurezza, promulgué par le régime fasciste, et uniquement soucieux de sécurité publique. Entre ces deux dates, les questions concernant la présence des étrangers en Italie n'étaient réglées que par circulaires administratives, ceci en contradiction avec la Constitution de 1947 qui stipule dans son article 2 que "la condition juridique de l'étranger est réglée par la loi en conformité avec les normes et traités internationaux" ( $c f$. article de F. Pastore). Le caractère discriminatoire des pratiques bureaucratiques en la matière a, entre faible contrôle des entrées et restriction des possibilités de séjours légaux, incontestablement contribué à alimenter la clandestinité de l'immigration, en relation avec la demande de l'économie souterraine.

La première manifestation d'une volonté politique d'intervenir sur la question de l'immigration est la loi du 30 décembre 1986 $\mathrm{n}^{\circ}$ 943, fruit des préoccupations des experts 


\section{L'enjeu migratoire, miroir de la crise politique italienne}

dans les réflexions qui entourent les Accords de Schengen, auxquels l'Italie veut adhérer (ce sera chose faite en 1990), mais aussi de la pression des associations humanitaires engagées dans des actions de solidarité avec les immigrés : d'un côté ce texte prétend instaurer une programmation des flux, de l'autre il s'agit d'une loi "garantiste" qui affirme pour la première fois les droits des immigrés (égalité de traitement et de droits des travailleurs extracommunautaires et des travailleurs italiens, droit à l'usage des services sociaux et de santé, droit à l'école, au logement, au maintien de l'identité culturelle, droit au regroupement familial). Cette loi inaugure ce qui va se révéler une pratique constante du système politico-administratif italien, celui de la régularisation des clandestins, qui, bien que nommée par les juristes regolarizzazione, est désignée par le langage courant, toujours révélateur, comme sanatoria (de sanare : guérir, assainir, couvrir).

Cette première émergence sur l'agenda politique est, certes, liée à une conjoncture. Le retour des attentats terroristes, notamment l'attentat de décembre 1985 à l'aéroport international $\mathrm{Da}$ Vinci à Rome, a été exploité à la fois pour illustrer l'inquiétude quant à la présence de plus en plus manifeste des étrangers, et pour dénoncer les dangers de la montée de la xénophobie. Mais il semble surtout y avoir eu là, pour le PSI, c'est-à-dire le gouvernement Craxi (1983-1987), alors à la tête de la coalition Pentapartito ${ }^{2}$, une occasion d'illustrer sa capacité à agir, une première tentative d'utilisation politique du thème. Il y a été poussé par un ensemble de groupes de pression transversaux aux partis et aux mouvements associatifs. Promulguée par le système des commissions parlementaires, cette loi a bénéficié de la coopération entre PCI (dans l'opposition) et DC (membre de la coalition au pouvoir), deux partis dont certains membres, jadis engagés sur les questions de l'émigration italienne, s'intéressent de près aux questions d'immigration. Ces personnalités politiques sont proches des milieux qui militent en faveur de l'insertion des immigrés, les syndicats confédéraux socialiste et communiste, et surtout les associations de volontaires catholiques autour du syndicat catholique et de son Comitato per una legge giusta pour les immigrés. De façon encore un peu incertaine, cette étape annonce les ingrédients permanents de la mise sur agenda du thème de l'immigration en Italie : un événement traumatisant, des tactiques partisanes, un travail de groupe de pression transversal aux clivages politiques qui préfigure une forme de consensus centriste qui caractérise toutes les lois sur l'immigration en Italie. Elle atteste aussi déjà d'un balancement négocié entre des mesures de contrôle, réclamées par les administrations et par les partisans de Schengen, et des mesures d'insertion et de régularisation, gages donnés aux groupes de pression favorables aux immigrés. Elle demeure en tout cas une affaire de spécialistes, militants ou experts, qui, si elle représente un problème collectif susceptible de traitement public, n'est pas encore un enjeu politique objet de stratégies.

Le véritable tournant législatif a, en fait, eu lieu avec la loi du 28 février 1990 n $^{\circ} 39$ (précédée d'un décret-loi), la très âprement discutée legge Martelli, loi considérée cette fois comme une mesure d'urgence. Celle-ci s'attache à régler, dans une perspective de sécurité publique, les conditions d'entrée et de séjour des étrangers, l'expulsion (sa principale caractéristique est de ne pas faire de l'entrée illégale un délit pénal, et de donner deux 


\section{Pôle Sud $N^{\circ} 11$}

semaines aux irréguliers, sans détention administrative, pour déposer un recours administratif contre leur ordre d'expulsion : ceux-ci en profitent évidemment pour repasser dans la clandestinité), la tutelle juridictionnelle, le statut de réfugié politique, l'inscription à l'état-civil, le travail indépendant et la programmation des flux. Elle réaffirme aussi la nécessité des politiques d'insertion. Elle institue enfin une deuxième vague de sanatoria.

La genèse de ce texte révèle parfaitement la complexité des processus décisionnels à l'cuvre. Durant l'été 1989, le meurtre dramatique d'un immigré, travailleur saisonnier agricole à Villa Literno au nord de Naples, considéré en raison de son destin tragique comme une figure emblématique de la misère du monde (Ritaine, 1998), déclenche un fort mouvement d'opinion favorable au respect des droits des immigrés : celui-ci est illustré par l'importante manifestation antiraciste du 7 octobre 1989, organisée par les nombreux groupes de solidarité avec les immigrés qui se sont créés dans le pays depuis quelques années. C'est donc en profitant de ce contexte de mobilisation antiraciste que le gouvernement démocrate-chrétien De Mita (coalition Pentapartito) s'attache à traiter une question que bloquaient jusqu'alors l'indifférence des politiques, voire l'hostilité des ministères de l'Intérieur (occupé par les Accords de Trevi sur le crime international et le terrorisme, donc soucieux de conserver le contrôle de l'immigration comme problème de sécurité publique) et des Affaires étrangères (attentif à ne pas compromettre les accords commerciaux avec les pays du Maghreb par l'imposition de l'obligation de visas pour les ressortissants de ceux-ci). Cette fois le dossier est confié à un leader socialiste important, viceprésident du Conseil, C. Martelli, dont la capacité d'influence se révélera décisive. Ce texte a reçu un soutien politique assez fort, et transversal aux partis, notamment parce que C. Martelli a recherché systématiquement l'accord des forces de gauche et qu'il a bénéficié du soutien du PCI. Les groupes de pression favorables à l'immigration se sont peu à peu laissés convaincre qu'une politique de non-restriction, telle qu'ils la souhaitaient, comporterait un sérieux risque de radicalisation des tensions xénophobes: il faut dire que le succès de la liste du Front national de J.-M. Le Pen aux élections européennes de 1984 sur une thématique xénophobe a beaucoup inquiété les forces progressistes en Italie, et que tous les mouvements démocrates sont attentifs à éviter à l'Italie ce risque de glissement xénophobe. Face à eux émerge, très nettement, l'influence des administrateurs de différents ministères dont la préoccupation centrale est de ne pas voir l'Italie exclue de l'espace européen en raison d'une politique trop laxiste vis-à-vis de l'Afrique du nord (ils obtiennent à ce titre l'obligation des visas pour tous les pays africains et maghrébins). Ce groupe de pression a sans doute été très actif dans la période qui sépare le décret-loi de sa transformation en loi, puisque toutes les modifications intervenues alors vont dans le sens d'un plus grand contrôle et d'une accentuation du rôle du ministère de l'Intérieur (Bolaffi, 1996; Veugelers, 1994).

Durant ce processus, la mobilisation différentielle des partis politiques atteste d'une incertitude quant aux effets politiques de l'exploitation de ce thème. Les grands partis, le PCI et la DC, interviennent rarement en place publique et travaillent plutôt dans l'aire parlementaire : sans doute est-il trop délicat pour eux de politiser un enjeu qui peut diviser leur électorat. Ce sont les petits partis qui ont 


\section{L'enjeu migratoire, miroir de la crise politique italienne}

tenté d'exploiter ce thème, cherchant, dans un système partisan très fragmenté, à maximiser l'avantage relatif qu'ils peuvent en retirer. Ceci décrit bien la tactique du PSI, mais aussi la logique des oppositions à un traitement politique trop favorable à l'immigration. La principale opposition à la loi Martelli est en effet venue, de façon surprenante, d'une des composantes de la coalition Pentapartito au pouvoir, le PRI. Se considérant comme la composante négligée de la coalition, et étant en forte concurrence avec le PSI, il a systématiquement fait obstruction sur le thème de la condamnation du laxisme gouvernemental, qui est un de ses slogans récurrents. Les autres oppositions viennent plus normalement de partis extérieurs à la coalition. Le MSI, parti néo-fasciste, est violemment opposé à cette loi et teste à cette occasion le développement de thèses xénophobes : cependant les risques de dissensions internes et les inconvénients en termes d'image pour un parti qui cherche à se dégager de son héritage fasciste (en 1994-95, le MSI se transformera en Alleanza nazionale, avec l'objectif d'être considéré comme un parti de la droite nationale respectueux des principes constitutionnels) l'empêchent de poursuivre sur cette pente; le thème xénophobe restera secondaire dans sa stratégie, son discours se portant plus volontiers sur la sécurité publique et sur l'efficacité de l'appareil d'État (Veugelers, 1994; Balbo, Manconi, 1990). La Ligue lombarde (la Ligue Nord, fédération des ligues septentrionales, ne sera créée qu'en 1991), quant à elle, bien qu'elle ne soit pas encore un acteur national influent (il faut pour cela attendre les élections législatives de 1992) est déjà puissante au plan régional et local : elle commence à se servir du thème de la crainte de l'immigration pour renforcer son slogan principal d'hostilité à l'État et à la classe politique, accusés de laxisme (Perlmutter, 1996; Diamanti, 1993).

On arrive ainsi au véritable tournant de la politisation du thème migratoire. En effet, si à la fin des années 80 plusieurs scandales liés à l'immigration ont défrayé la chronique (l'agression de Villa Literno en 1989, la "ratonnade" de la nuit du Mardi gras à Florence en 1990, la découverte la même année d'un immense et misérable squat d'immigrés clandestins au centre de Rome, la Pantanella) (Ritaine, 1998; Gallini, 1991), ils ont en général abouti à des mobilisations antiracistes, comme la manifestation à Rome en octobre 1989, ou au moins à une vague de commisération dans la société italienne. Révélatrice d'une prise de conscience collective, la Première conférence nationale sur l'immigration s'est tenue en juin 1990, en présence de deux mille délégués et des représentants de deux cent cinquante associations d'immigrés. Les premiers exodes albanais eux-mêmes, pourtant spectaculaires (notamment celui de mars 1991 qui amène 21000 personnes sur la côte de Bari), sont assez bien tolérés par la population méridionale, qui fait preuve de solidarité, et grâce à l'action des associations humanitaires. Ceci malgré un durcissement du gouvernement qui, au deuxième exode en août, passe brutalement de la passivité à la répression, regroupe les Albanais dans le stade de Bari et expulse manu militari une grande partie d'entre eux. Passé cet épisode dramatique, et son traitement spectaculaire par la presse, la question migratoire disparaît de l'agenda politique et des débats publics (Bonifazi, 1998).

L'Italie vient en effet d'entrer dans la plus grave crise politique de l'après-guerre : l'ensemble du système politique italien s'est effondré très brusquement dans les scandales, 


\section{Pôle Sud $N^{\circ} 11$}

celui du Gladio en 1990, ceux que dévoile sans cesse l'opération Mani pulite à partir de 1992, les soubresauts de la lutte contre la mafia depuis le début des années 90. Tous les grands partis, dont on avait pu dire qu'ils étaient la véritable centralité italienne, sombrent ou se transforment décisivement; cependant que de nouveaux acteurs, véritables entrepreneurs de la crise, apparaissent pour modifier le jeu (Ligue, Forza Italia). Le système politico-administratif est secoué par un véritable tremblement de terre, où les questions essentielles sont celles de la réforme institutionnelle et de la moralisation de la vie publique. Quant aux citoyens italiens, ils sont pris entre, d'une part, un scepticisme généralisé quant à la norme publique, qui peut les pousser vers les partis extrêmes ou protestataires, et, d'autre part, l'engagement dans la recherche d'un consensus politique rénové. Centrée sur elle-même, la société italienne ne s'interroge plus guère sur la figure de l'Étranger. Dans ce silence du politique, les effets des incidents xénophobes, qui se multiplient, dont la presse fait écho sur un mode sensationnel, et que la Ligue récupère avec délectation, sont dévastateurs. L'année 1993 est particulièrement agitée de ce point de vue : nouvelles violences à Villa Literno, émeutes dans le centre de Gênes, rondes contre les marchands ambulants sur la côte Adriatique sous l'égide de la Ligue nord, etc. Profitant du désarroi lié à la crise politique, les thèses d'extrême droite, taboues depuis la chute du fascisme, s'affichent en effet ouvertement. Aux élections municipales de 1993, deux partis, le MSI et la Ligue, développent explicitement le thème de "l'urgence immigration" (emergenza immigrazione), naturalisant ainsi la thématique de l'insécurité urbaine liée à l'immigration qui va devenir dominante quelques années plus tard, et dans un contexte défavorable aux forces de gauche, en tirent quelques avantages électoraux. À partir de là, cette interprétation sécuritaire de l'immigration deviendra dominante et obligera tous les acteurs à se situer par rapport à elle. Ceci est manifeste dès que la coalition de droite (le Pôle des libertés composé de Forza Italia, la Lega Nord et Alleanza nazionale, ex-MSI) remporte les élections législatives de 1994. Sous la pression de ces partis, et dans le contexte humiliant de l'exclusion de l'espace Schengen, le gouvernement de techniciens de L. Dini doit prendre plusieurs mesures rigoureuses qui entérinent la dimension restrictive et répressive de la politique italienne : le déploiement des forces armées dans les Pouilles, pour tenter de limiter le trafic de clandestins en provenance de ou en transit par l'Albanie, et surtout le décret-loi de 1995 durcissant les modalités d'expulsion (pour motif de clandestinité ou pour délit chez les immigrés en situation régulière) et organisant une nouvelle sanatoria.

\section{In-sécurité nationale : au coeur de la crise politique}

Texte décisif en ce qu'il instaure pour la première fois la stigmatisation de l'immigré comme problème d'ordre public, et qu'il contraint tous les acteurs politiques, y compris la gauche, à se définir par rapport à l'intérêt national face à ce péril politiquement construit : la "pensée d'État" s'instaure (Sayad, 1996).

Période décisive aussi en ce qu'elle est marquée en Italie par un débat sur l'intégration nationale et que la question migratoire peut alors avoir une "fonction-miroir" (Sayad, 1990). La crise politique italienne des 


\section{L'enjeu migratoire, miroir de la crise politique italienne}

années 90 est en effet à la fois une mise en crise de la norme étatique et un malaise de l'intégration nationale (Ritaine, 1994). Le désenchantement des citoyens face à l'État y a été remarquablement exploité par les "entrepreneurs politiques de la crise" (Diamanti, 1993), qui, bien que dans des sens différents, ont interprété la crise en termes de problèmes d'intégration nationale. Au premier rang d'entre eux, la Ligue et son discours "populiste régionaliste" (Biorcio, 1991) : ce parti code le mécontentement des citoyens sous la forme d'une contestation radicale de l'existence d'un État-nation italien et d'une revendication de l'autonomie des territoires du nord de l'Italie dans une solution fédérale. La légitimation de cette appartenance politique réactive nécessite la désignation symbolique de figures sociales antagonistes (Dal Lago, 1999) : l'État désigné comme "romain" et "voleur" tout d'abord, les régions méridionales considérées comme parasitaires ensuite, les immigrés extracommunautaires (qu'on désignera alors comme arabi ou africani) enfin. On condamne ainsi, en des raccourcis saisissants, "la méridionalisation de l'État central et l'africanisation de la cité" (Manconi, 1990, p. 72), dans des campagnes xénophobes de plus en plus violentes. “Dans les années 90, la Ligue s'est signalée comme la formation la plus engagée dans la propagande et la mobilisation contre les immigrés extracommunautaires, les Gitans et la microcriminalité [...]. L'engagement de la Ligue sur ce terrain s'est exprimé par l'organisation de "rondes" destinées à protéger les populations et les cités du Nord de l'établissement des extracommunautaires et des Gitans, et, plus récemment, de l'arrivée des Albanais. Même si elles sont sans effets pratiques, ces initiatives et ces polémiques ont influencé l'opinion et les attitudes populaires, et ont créé une forte symbolique pour le mouvement lui-même" (Biorcio, 1997, p. 270). A l'opposé de la demande fédéraliste de la Ligue, Alleanza nazionale (ex-MSI), alliée de la Ligue au sein du Pôle des libertés, milite pour la rénovation de la nation italienne et pour la réhabilitation de l'autorité de l'État. Aussi adopte-t-elle des positions plus centrées sur la défense de la communauté nationale et prône-t-elle, dans un discours de la responsabilité publique censé l'éloigner de son passé néofasciste, le durcissement des mesures de contrôle des frontières et d'expulsion des clandestins.

La thématique développée par ces deux acteurs politiques autour de l'idée nationale a contraint tous les autres à se définir sur ces thèmes, notamment les partis de gauche en marche vers leur destin de partis de gouvernement. Le débat autour des fondements et du destin de l'identité nationale a été intense ces dernières années : dans ce contexte, la dichotomie entre nationaux, citoyens, légitimes, d'une part, et étrangers, non citoyens, illégaux, d'autre part, remplit une fonction symbolique forte. Sur ce mode, le pan restrictif et répressif de la politique d'immigration, renforcé par les normes Schengen, attisé par les campagnes xénophobes, devient incontournable pour tous les acteurs politiques.

\section{Une "pensée d'État"? Au miroir de Schengen}

Cette évolution est très apparente depuis la victoire électorale du centre-gauche en 1996. Le nouveau gouvernement de R. Prodi a en effet dû affronter en 1997 des crises successives liées aux questions migratoires. Un nouvel exode albanais tout d'abord, qui l'a amené à instituer le blocus de la côte Adriatique par 


\section{Pôle Sud $N^{\circ} 11$}

la marine italienne et la participation italienne à l'envoi d'une force multinationale en Albanie. L'arrivée massive de réfugiés kurdes, dans le courant de l'hiver, qui a obligé à maintenir le contrôle aux frontières terrestres avec la France et l'Autriche. Lintensification surtout de la campagne xénophobe de la Ligue, relayée par l'ensemble des partis de droite, tout au long de l'année, de plus en plus axée sur le thème de l'insécurité urbaine (incidents et manifestations à Turin et Milan notamment) : les représentations de la "dangerosite" liée à l'immigration, de plus en plus fréquemment rapportée aux risques de la délinquance et au risque mafieux, se sont à l'évidence naturalisées (Dal Lago, 1999³). Ce contexte, mais aussi la décision de ses partenaires européens de finalement accepter l'inclusion de l'Italie dans l'espace Schengen à condition qu'elle se mette en conformité avec les normes européennes, a accéléré la présentation de la nouvelle loi sur l'immigration ${ }^{4}$ et marqué son esprit, désormais fortement sécuritaire.

Il est tout à fait révélateur que la loi du 6 mars 1998, $n^{\circ} 40$, porte le double nom de Turco-Napolitano, la première étant ministre de la Solidarité nationale, le second ministre de l'Intérieur, et tous deux membres du PDS : on ne peut donc douter que le projet de loi ait représenté la position ambivalente, contrôle et intégration, du centre-gauche sur ces questions. L'aspect policier et restrictif de la loi est manifeste, puisqu'y sont confirmées la politique d'expulsion des clandestins avec l'instauration de centres de séjour temporaire à laquelle l'Italie avait été longtemps réticente, et la politique de contrôle des quotas de travailleurs immigrés. La volonté d'intégration s'est elle aussi renforcée, avec l'affirmation de l'égalité des droits entre Italiens et immigrés réguliers (droits à l'assistance sanitaire, au loge- ment social, obligation scolaire pour les enfants, etc.), même si le rejet de l'article qui prévoyait le droit de vote passif et actif pour les immigrés réguliers aux élections locales finira par limiter la portée innovatrice de ce texte.

Les conditions d'adoption de cette loi sont révélatrices de la nature du consensus politique qui règne sur ces questions (Fondazione Cariplo, 1999). Dans un système de partis très fragmenté, le gouvernement a dú tenir compte de deux formes d'opposition. En dehors de la Ligue, qui, fidele à sa ligne xénophobe, a plutôt choisi de jouer l'obstruction systématique, l'opposition de droite vient d'Alleanza nazionale et de Forza Italia, qui soutiennent les mesures les plus restrictives, mais refusent absolument le droit de vote des immigrés aux élections locales. Plus dangereuse pour le projet gouvernemental est l'opposition de gauche, celle des Verdi et de Rifondazione comunista, qui refusent les articles trop répressifs du projet (en particulier les centres de rétention temporaire) et sont favorables aux mesures d'intégration (et surtout à l'attribution du droit de vote). Compte tenu de ces tensions, le gouvernement ne peut obtenir une majorité que par un compromis parlementaire. Il donnera donc des gages à l'opposition de droite en retirant au dernier moment l'article prévoyant le droit de vote aux élections locales, et à l'opposition de gauche en créant un permis de séjour pour recherche d'un emploi et en complexifiant tellement les mesures de répression de la clandestinité qu'il restera souvent une façon d'éviter la reconduite à la frontière. Une nouvelle régularisation complète le dispositif'. Aussi le texte final est-il une solution de compromis entre la répression de l'immigration clandestine et des mesures d'intégration sociale des immigrés réguliers, qui met formellement 


\section{L'enjeu migratoire, miroir de la crise politique italienne}

l'Italie en conformité avec les normes européennes. On aboutit ainsi à un résultat assez semblable aux politiques définies par les autres pays européens, une solution moyenne entre effort de contrôle et volonté d'intégration.

Loriginalité de la situation italienne tient beaucoup plus aux conditions politiques du débat, la longue incohérence de l'action publique ayant laissé l'espace symbolique disponible pour une exploitation xénophobe, qui a imposé une interprétation sécuritaire. Ce vide politique est certes créé par le fonctionnement au compromis au niveau des échanges partisans. Il l'est aussi par les caractéristiques du style politique (au sens de policy style) italien, qui tend à des prises de décision réactives à l'urgence, et à une mise en application faible, mais aussi à la multiplication de médiations politiques alternatives. Envisagées de ce point de vue plus structurel, les conditions du débat sur l'immigration se complexifient, notamment par l'entrée en scène de nouveaux acteurs.

"Des urgences continues et permanentes surgissent dans presque tous les secteurs de l'activité politique et sont utilisées, avec une efficience décroissante, pour forcer à une décision significative des mécanismes qui ne semblent pas capables d'en produire. Qu'il s'agisse des déchets toxiques ou de l'implication militaire au Moyen-Orient, de l'effondrement de quelque monument ou de la recrudescence de la criminalité mafieuse, les institutions italiennes semblent constamment anxieuses, à la recherche de solutions provisoires qui réussissent quelque peu à colmater la crise. Parfois, comme par exemple dans le cas du terrorisme, le recours à la solution d'urgence réussit à porter ses fruits. Dans d'autres cas, au contraire, le caractère structurel des problèmes empêche de tirer bénéfice de la dramatisation. Ce qui semble plus grave, en termes d'evaluation, est qu'il $y$ ait des politiques qui entrent bien difficilement dans cette crise verticale qui peut justifier l'urgence, et qu'en conséquence elles soient décidées presque uniquement à travers des décisions de petit cabotage, prises en réponse à quelque événement extérieur." (Dente, 1990, p. 43-44). Jusqu’à présent, la politique de l'immigration s'est bien constituée de réponses successives aux urgences : urgence européenne, urgence de l'utilisation politique des thèmes xénophobes, urgence des événements extérieurs.

Ce sont les pouvoirs politiques locaux, municipalités et régions, qui, devant faire face à des situations locales tendues, ont pratiqué le "petit cabotage", en tentant d'inventer des politiques d'insertion sociale. En effet, c'est au niveau local que les tensions sociales ont exigé le plus de réactions politico-administratives : la présence extracommunautaire, et notamment la présence des immigrés en situation précaire, est en effet fortement concentrée dans les zones urbaines (notamment à Rome, Milan, Naples, Turin, Florence, Vicenza, Palerme). Cette sururbanisation du phénomène est à la fois un facteur de marginalisation des immigrés et un facteur de plus grande visibilité sociale de la présence immigrée. Ce sont donc le plus souvent les administrations municipales qui ont dâ prendre en charge les problèmes urgents, ceux du logement, de l'assistance sanitaire et sociale, de l'aide à l'insertion dans le marché du travail. Ce faisant les autorités locales ont fait preuve de capacités extrêmement différentes : en partie parce que l'absence d'un référentiel national fort laisse flotter les initiatives au gré des urgences et des ressources et inhibe les capacités de planification à moyen terme; en partie parce que la capacité organisationnelle des 


\section{Pôle Sud $N^{\circ} 11$}

pouvoirs locaux est très différenciée sur le territoire, en fonction des contextes socioculturels, des traditions politico-administratives, des ressources économiques, des capacités de mobilisation (Fondazione Cariplo, 1995). La capacité à prendre en charge un problème d'intérêt général s'est, sur cette question comme sur bien d'autres (Ritaine, 1995, 1997 ; Putnam, 1993), révélée plus forte dans les régions du Centre et du Nord qu'au Sud : cette inégalité de traitement public redouble ainsi la différenciation territoriale de l'immigration, qui tend à être plus récente et plus précaire au Sud, plus stabilisée et en voie d'insertion vers le Nord. En toute logique, les politiques locales ont été dans le meilleur des cas des politiques de protection de la société locale vis-à-vis de potentiels déviants (elles ont alors pour acteurs centraux les agents du maintien de l'ordre), ou des politiques d'assistance qui traitent l'immigration comme un nouveau genre de pauvreté (elles sont plutôt le fait des services sociaux) (Allasino, Baptiste, Bulsei, 1995) (cf. article d'E. Allasino).

\section{Des médiateurs dans la société civile}

La faiblesse de la mise en ouvre des politiques migratoires par l'appareil politicoadministratif révèle, par contraste, l'importance d'autres acteurs, qui apparaissent non seulement comme des maîtres d'œuvre, mais aussi comme de puissants inspirateurs des politiques elles-mêmes. État comme pouvoirs locaux se déchargent en fait de la gestion de la présence immigrée sur le "troisième secteur ${ }^{6 \text { ", }}$ entérinant ainsi le classique éclatement du Welfare state italien. Aussi est-ce, in fine, dans la société civile qu'il faut chercher les acteurs principaux de la prise en charge de la gestion de la présence immigrée. "Le troisième sec- teur" est en effet un facteur structurant du débat sur l'immigration, tant en termes d'organisation qu'en termes de définition : il apparaît comme un ensemble de médiateurs politiques fondamentaux sur ces thèmes, car non seulement en charge de la gestion pratique de la question, mais aussi en position d'influer sur la définition des politiques, et sur les termes du débat.

Au premier rang de ces médiateurs, les réseaux du catholicisme le plus social, aux engagements missionnaires, qui assimilent tiers-mondisme et immigration. Ces réseaux étaient déjà à l'origine, depuis longtemps, de courants d'immigration catholique organisé, en provenance des Philippines, de l'Amérique latine, du Cap Vert, d'Inde et du Sri Lanka, d'Éthiopie-Erythrée, de Somalie, etc. : c'est ainsi que les couples de Philippins ou les femmes éthiopiennes, engagés comme employés de maison, sont devenus des figures sociales familières en Italie. Ces réseaux sont désormais fortement engagés dans des actions de solidarité avec les immigrés, sur la base du volontariat catholique, et d'une lutte contre la "nouvelle pauvreté". Il s'agit là de la plus forte présence sociale sur ce terrain, puisqu' environ les deux tiers des organisations de volontaires italiens sont d'origine catholique. Ces organisations sont plus nombreuses dans la partie nord du pays, terres de tradition catholique, et manifestent "l'attention persistante du monde ecclésial pour les formes émergeantes de marginalité et l'orientation ouvertement favorable au pluralisme ethno-culturel de cette partie de l'associationnisme social" (Fondazione italiana per il volontariato, cité in Fondazione Cariplo, 1995, p. 133). L'importance des organisations paroissiales, et d'associations comme San Egidio et surtout Caritas, en fait de véritables substituts à l'action 


\section{L'enjeu migratoire, miroir de la crise politique italienne}

publique, défaillante en fait d'insertion : en 1991, l'Église gérait 4700 centres d'assistance sociale, soit en moyenne 21 par diocèse, et il est probable que ce chiffre ait augmenté depuis (Pace, 19987). L'associationnisme laïque de son côté, articulé au travail des syndicats de gauche qui ont toujours intégré les travailleurs immigrés en leur sein (et qui vont souvent jusqu'à être des recours pour les travailleurs clandestins), est de plus en plus présent sur le terrain, animant de nombreuses associations antiracistes et collaborant avec les municipalités de gauche. Ce sont ces groupes, ainsi que les pouvoirs locaux, qui codent la question de l'immigration en termes de question sociale et qui travaillent à l'insertion sociale des populations immigrées. Nouvelle pauvreté à laquelle s'applique le souci caritatif des catholiques d'un côté, nouveaux exclus que les forces de gauche doivent défendre de l'autre : il sera intéressant d'observer si les deux interprétations, universalistes, divergent ou se complètent à l'avenir. En effet, ces deux tendances du mouvement social sont peut-être des formes de redéfinition de l'engagement social en Italie, et elles sont en ce sens en concurrence. L'engagement catholique semble y trouver une voie de renouvellement après les années de désillusion qui ont suivi le naufrage de la démocratie chrétienne, et alors que l'Église ambitionne visiblement de redevenir un recours éthique pour la société italienne (Cartocci, 1994). Le militantisme lä̈que y trouve peut-être aussi une de ses nouvelles voies, parallèlement aux engagements écologistes, féministes, pacifistes, dans la recomposition qui se dessine depuis la transformation du PCI en parti de centre-gauche et de gouvernement (Caciagli, 1995).

Ces réseaux ne sont pas seulement des instances organisationnelles, substitutives du ser- vice public. Ils apparaissent aussi, surtout, comme de puissants acteurs dans la définition des termes du débat, et, bien que leur rôle soit encore peu étudié, il est possible que leur capacité d'influence soit un élément décisif dans les années à venir. En 1986 déjà, la pression exercée par le Comitato per une legge giusta, auquel participaient associations catholiques et syndicats confédérés avait marqué profondément la loi de 1986. Cette forme d'influence s'était répétée dans les années 90 avec le pacte Nuovi diritti di cittadinanza per un Parlamento antirazzista, dont faisaient partie à la fois des associations chrétiennes (ACLI-Associazione cristiane dei lavoratori italiani -, Caritas, Federazione degle chiese evangeliche italiane, etc.), et des associations de gauche (ARCI - Associazione ricreativa culturale italiania -, Coordinamento immigrati CGIL, Italia Razzismo, etc.). Ce rassemblement demandait aux futurs députés et sénateurs d'adhérer à ce pacte et de soutenir ensuite l'application de ses principes dans la production législative. La loi la plus récente, celle de 1998, est aussi le fruit de négociations serrées et longues, encore que peu connues, entre représentants du gouvernement et syndicats et associations : ce sont ces réseaux qui fournissent les experts consultés. De plus, avec les deux gouvernements de centre-gauche, dont la majorité va des Verdi et des DS (exPDS) aux catholiques de centre-gauche, l'influence est souvent directe : aujourd'hui, dans le gouvernement D'Alema, le portefeuille de l'Intérieur est détenu par une ministre catholique du PPI, R. R. Jervolino ${ }^{8}$, et celui de la Solidarité sociale par une ministre d'origine communiste, L. Turco, des DS.

Désormais, le lobby pro-immigration, puisque tel est le terme en vogue en Italie, a un accès direct aux cercles de la décision poli- 


\section{Pôle Sud $N^{\circ} 11$}

tique. Il a donc quelques difficultés à maintenir une distance critique, et semble lui aussi avoir intégré la thématique sécuritaire. Aujourd'hui, lorsque ces réseaux de militants critiquent les insuffisances dans l'application de la loi, ils le font en manifestant qu'eux aussi ont intégré l'interprétation dominante : Caritas reproche ainsi au gouvernement un traitement incohérent de l'immigration illégale qui multiplie les risques de discrimination entre clandestins et qui favorise la montée de la xénophobie ; cependant que le monde syndical et l'extrême-gauche, descendant dans la rue pour s'opposer à la montée des thèmes xénophobes, par exemple pour répondre à la manifestation "tolérance zéro" du Pôle, se mobilisent sur le thème d'une cité "sûre et solidaire ${ }^{10 "}$. Plus aucun des acteurs de la construction politique de l'enjeu migratoire ne semble avoir de doute sur la dangerosité de l'immigration : une interprétation dominante, un "style public de définition de la réalite" (Dal Lago, 1999, p. 123) se sont imposés. Reste que, si cette interprétation a des effets pratiques autant que symboliques, et si elle constitue le cadre cognitif du débat, son analyse devra être complétée par la connaissance des pratiques sociales autour du phénomène migratoire. Celle-ci ne peut s'élaborer que sur une étude des pratiques des acteurs mobilisés autour des thèmes migratoires, et sur une recherche sur les processus d'acculturation réciproque. Une sociologie de l'action des médiateurs en particulier (associations catholiques, associations laiques, associations d'immigrés, experts ministériels) devrait permettre d'avancer sur les processus sociaux (et non plus seulement politiques) à l'œuvre dans la confrontation à l'altérité: sans doute les thématiques de demain y sont-elles en gestation.

Le contexte de profonde recomposition politique en Italie permet de comprendre comment a pu s'ouvrir un tel espace à l'exploitation sécuritaire, xénophobe donc au sens étymologique du terme, d'un phénomène social somme toute limité. Tout au long des années 90, la demande des citoyens italiens s'exprime en termes d'efficience de l'appareil d'État, de respect de la légalité, de moralité publique, de sécurité collective. Comment ne pas voir que, de même que le politicien potentiellement corrompu, de même que le méridional potentiellement mafieux, l'immigré potentiellement délinquant est une figure idéale du sentiment d'in-sécurité nationale? D'autant que lui-aussi, comme les deux autres figures, renvoie l'imaginaire à ses interrogations sur l'appartenance nationale : "Si nous cessons d'être une nation" (Rusconi, 1993), comment ne pas considérer l'Autre comme une étrangeté menaçante? Et, si nous ne savons à quelle communauté l'intégrer, ou de quelle communauté l'exclure, comment coder cette présence autrement qu'en termes sécuritaires? Le danger de glissement xénophobe est dans cette béance. Le discours politique n'a finalement fait que décalquer les normes européennes, en produisant, avec difficulté, un consensus entre répression et intégration. Seul le populisme régionaliste a tenu un discours puissant, sur le thème du danger migratoire, et a ainsi déterminé les termes du débat en imposant l'équivalence immigration-insécurité. Lorsque le PDS lui-même organise en 1997 Vivere sicuri, un forum européen sur la sécurité/insécurité urbaine où la question de l'immigration revient comme un leitmotiv ${ }^{11}$, la boucle est bouclée : quels acteurs désormais penseront l'interculturalité, le renouvellement de la citoyenneté, les tensions entre ethnos et demos? 


\section{L'enjeu migratoire, miroir de la crise politique italienne}
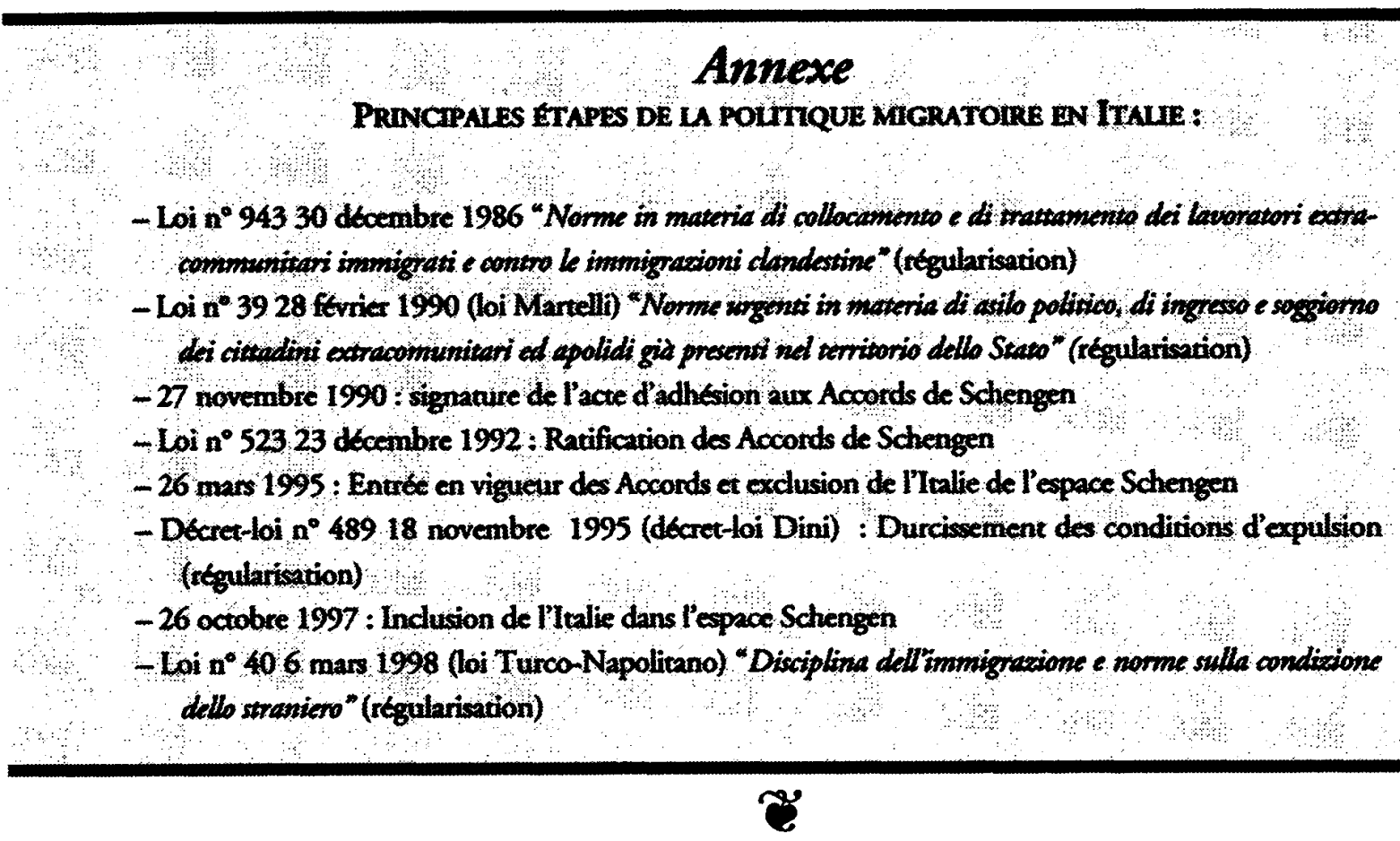

Notes

1. L'Italie adhère aux Accords de Schengen (1985) en 1990, et les ratifie en 1993. A l'entrée en vigueur de ces accords, en 1995, cependant, ses partenaires décident de l'exclure de l'espace Schengen. Deux raisons sont avancées à cela : l'Italie ne dispose pas de la protection des données personnelles nécessaires pour participer au SIS (Système Information Schengen) qui centralise toutes les données nécessaires au contrôle des étrangers entrant dans l'espace de libre-circulation européen; l'Italie n'est pas considérée comme capable de contrôler les flux d'arrivée à ses frontières. En 1997, au moment de la signature du Traité d'Amsterdam et grâce à une intense campagne diplomatique du gouvernement Prodi, l'Italie est incluse dans l'espace Schengen. La condition est qu'elle se mette en conformité avec les normes Schengen, notamment en votant avant avril 1998, une loi générale sur l'immigration : ce sera, in extremis, la loi $n^{\circ} 40$ du 6 mars 1998.

2. De 1981 à 1992, l'Italie a été gouvernée par une coalition de cinq partis, dite Pentapartito: DC, PSI, PSDI, PRI, PL.

3. A. Dal Lago (1999) relève avec inquiétude que cette équivalence a atteint jusqu'à la recherche en sciences sociales qui se préoccupe désormais de la "propension" à la délinquance parmi les immigrés, ignorant par là même les effets de sélection discriminante du système pénal lui-même. Pour une analyse critique, voir Palidda, 1994, 1996.

4. "S'était constituée (depuis l'arrivée du nouveau gouvernement Prodi) une commission gouvernementale comprenant quelques experts afin de préparer un projet de loi générale qui deviendrait, en Commission des affaires constitutionnelles, le texte de base. Le projet de loi fut présenté en février 1997, mais la discussion en commission commença seulement à la fin du mois de mai. Le parcours parlementaire en Commission des affaires constitutionnelles fut soudain interrompu en septembre 1997. A un mois de la réunion européenne qui devait entériner l'entrée de l'Italie dans l'espace Schengen, selon une procédure inhabituelle, la discussion du texte se poursuivit à partir de l'article 12 en assemblée, et en quelques séances remarquablement longues, la Chambre vota un texte qui devait être approuvé sans modification par le Sénat en mars 1998" (Fondazione Cariplo, 1999, p. 63). 


\section{Pôle Sud $N^{\circ} 11$}

5. Initialement prévue pour 38000 personnes, elle en concernera finalement, vu l'ampleur de la demande, 250000 (début 1999).

6. On reprend ici la terminologie courante en Italie pour désigner les organisations "nonprofit", i.e. des organisations privées sans but lucratif qui remplissent des fonctions d'utilité publique, qui ont un minimum de structuration et qui utilisent des intervenants volontaires. Il s'agit d'une piste de réflexion importante dans de futures analyses des enjeux migratoires.

7. Ceci n'étant pas exclusif de fortes tensions internes au clergé italien, les diocèses du Sud par exemple se montrant plus tolérants à l'égard de l'immigration, que ceux du Nord et du Nord-Est, souvent tentés par des positions proches de celles de la Ligue.

8. Celle-ci, titulaire du ministère des Affaires sociales, au titre de la DC, dans le gouvernement De Mita en 1988, était normalement chargée de la préparation de la loi sur l'immigration. Les réticences des partis et de l'appareil d'État obligèrent le gouvernement à confier le dossier à un responsable politique très influent, G. Martelli, dont la loi de 1990 porte le nom (Veugelers, 1994).

9. Conférence sur les évaluations de Caritas en matière d'immigration pour 1999, 27 février 1999, in La Rocca O. : "La Caritas accusa il governo : 'sugli immigrati ha fallito'", La Repubblica, 28 février 1999.

10.Bonerandi E. : "Sfila l'altra Milano, in piazza per una città "sicura e solidale", La Repubblica, 14 février 1999. 11. “Il PDS di governo scopre la repressione”, La Repubblica, 23 mars 1997, cité par Dal Lago, 1999, p. 138.

\section{Références}

Allasino E., Baptiste F., Bulsei G.L., “Gli incerti confini : politiche per gli immigrati e politiche di lotta all'esclusione sociale a Torino e Lione", Polis, $\mathrm{n}^{\circ} 1,1995$.

Balbo L., Manconi L., (eds.), I razzismi possibili, Milan, Feltrinelli, 1990.

Balbo L., Manconi L., (eds.), I razzismi reali, Milan, Feltrinelli, 1992.

Biorcio R, La Padania promessa. La storia, le idee e la logica d'azione delle Lega nord, Milan, Il Saggiatore, 1997.

Bolaffi G., Una politica per gli immigrati, Bologne, Il Mulino, 1996.

Bonifazi C., L'immigrazione straniera in Italia, Bologne, Il Mulino, 1998.

Caciagli M., "La destiné de la 'subculture rouge' dans le centre-nord de l'Italie", Politix, n 30, 1995.

Cartocci R, Fra Lega e Chiesa. L'Italia in cerca di integrazione, Bologne, Il Mulino, 1994.

Dal Lago A., Non-persone. L'esclusione dei migranti in una societa globale, Milan, Feltrinelli (Interzone), 1999.

Dente B., Le politiche pubbliche in Italia, Bologne, Il Mulino, 1990.

Diamanti L., La Lega : geografia, storia e sociologia di un nuovo sogetto politico, Rome, Donzelli, 1993.

Fondazione Cariplo, Primo rapporto sulle migrazioni 1995, Milano, Angeli, 1995.

Fondazione Cariplo, Quarto rapporto sulle migrazioni 1998, Milano, Angeli, 1999.

Gallini C., "Mises en scène du racisme italien", Terrain n' 17 : "En Europe les nations", 1991.

Mannheimer R. (ed.), La Lega lombarda, Milan, Feltrinelli, 1991.

Pace E., La nation italienne en crise. Perspectives europeennes, Paris, Fayard, 1998.

Palidda S., Devianza e criminalità tra gli immigrati, Milan, Fondazione Cariplo/Ismu, 1994.

Palidda S., Verso il "fascismo democrativo" ? Note su emigrazione, immigrazione e società dominanti, aut aut, 275, 1996.

Perlmutter T., "Immigration Politics Italian Style : The Paradoxical Behaviour of Mainstream and Populist Parties", South European Society and Politics, I, n' 2, 1996.

Putnam R. D. (ed.), Making Democracy Work. Civic Traditions in Modern Italy, Princeton, Princeton University Press, 1993. 


\section{L'enjeu migratoire, miroir de la crise politique italienne}

Ritaine E., "Territoire et politique en Europe du sud", Revue française de science politique, $\mathrm{n}^{\circ} 1,1994$.

Ritaine E., "Italie : territoires au bord de la crise politique", in Balme R., Garraud P., Hoffmann-Martinot V., Ritaine E., (dir.), Le territoire pour politiques : variations européennes, Paris, L'Harmattan, 1994.

Ritaine E., "La capacité politique des régions en Europe du sud", in Le Galès P., Lequesne C., (dir.), Les paradoxes des régions en Europe, Paris, La Découverte, 1997.

Ritaine E., "La barrière ou la mémoire? Construction sociopolitique de l'enjeu migratoire en Italie", in Ritaine E. (dir.), Le territoire au miroir de litinéraire, Bordeaux, Cahiers du CERVL, Rapport de recherche 4, 1998.

Rusconi G.E., Se cessiamo di essere una nazione, Bologne, Il Mulino, 1993.

Sayad A., L'immigration ou le paradoxe de l'alterité, Bruxelles, De Boeck-Wesmael, 1990.

Sayad A., "La doppia pena del migrante. Riflessioni sul 'pensiero di stato" ", aut aut, 275, 1996.

Veugelers J. W. P., "Recent Immigration Politics in Italy : a Short Story", West European Politics, n 2, 1994.

Zincone G., Uno schermo contro il razzismo. Per una politica dei diritti utili, Rome, Donzelli, 1994.

\section{Résumé/Abstract}

L'originalité de l'Italie, dans l'ensemble sud-européen, réside tout d'abord dans la politisation du thème de l'immigration. Cette politisation se réalise aussi dans un sens spécifique : non seulement on y retrouve le balancement habituel entre nécessités répressives et volontés démocratiques, mais encore voit-on s'y réaliser, par l'entremise de certains partis politiques, une "naturalisation politique" des thèmes xénophobes, sous la forme d'une équivalence symbolique entre insécurité et immigration. La dynamique des prises de position, dans les rapports interpartisans et dans la société civile, est prise dans le mouvement de la recomposition politique italienne. Ce contexte rend, plus qu'ailleurs, sensibles les effets de la confrontation à l'altérité sur les représentations de la Nation et de l'État, de la nationalité et de la citoyenneté, qui sont au principe de la crise politique italienne.

Within southern Europe, the specificity of the Italian approach to immigration is its degree of politicisation. This has also taken a specific course : not only is there the usual balancing act between repressive necessities and commitments to democracy, but, through certain political parties, there has been a natuaralisation of xenophobic themes which has taken the form of equating insecurity with immigration. The dynamics of this positionning, both in terms of inter-party relationships and in civil society, is caught up in the ongoing recomposition of Italian politics. More than in other countries, this context has made the effects of confronting the Other even stronger on representations of the nation, the state, nationality and citizenship, concepts which are all at the heart of the Italian political crisis.

\section{Mots-clés/Keywords}

Politique migratoire, parti politique, médiation politique, xénophobie, Italie Migration policy, political party, political mediation, xenophobia, Italy 